\title{
LA UTILIDAD DE CONOCER EL NOMBRE DE LAS LETRAS PARA ADQUIRIR EL SISTEMA DE ESCRITURA $^{1}$
}

\section{GIULIANNY RUSSO MARINHO}

Universidad Nacional de La Plata - Argentina. Magíster en Escritura y Alfabetización de la Universidad Nacional de La Plata - Argentina. Especialista en Alfabetización por el Centro de Formación Escola da Vila. Pedagoga pela Universidade de São Paulo - USP. Maestra de nível inicial y grados iniciales. ORCID 0000-0002-1157-8364. E-mail: giulianny.russo@gmail.com

\section{MÓNICA ALVARADO}

Universidad Autónoma de Querétaro - México. Doctora en Ciencias con Especialidad en Investigación Educativa. Profesora da la Facultad de Psicología de la Universidad Autónoma de

Querétaro - México. ORCID 0000-0001-9847-9061. E-mail: monicaalvarado@yahoo.com

1 En este trabajo presentamos resultados de una investigación realizada en el marco de la Maestría de Escritura y Alfabetización de la Universidad de La Plata, Argentina. Disponible en http://www.memoria.fahce.unlp.edu.ar/tesis/ te.1711/te.1711.pdf 


\section{LA UTILIDAD DE CONOCER EL NOMBRE DE LAS LETRAS PARA ADQUIRIR EL SISTEMA DE ESCRITURA}

La investigación desde la psicología cognitiva señala que el conocimiento del nombre de la letra es esencial para aprender a leer y escribir. Sin embargo, la investigación psicogenética muestra que el uso de la información (nombre de la letra) está condicionado por la forma en que los niños comprenden el funcionamiento del sistema de escritura. El objetivo de este trabajo fue investigar la relación entre identificar letras por sus respectivos nombres y la posibilidad de usarlas de manera pertinente en contextos de escritura y completar palabras, observando la influencia ejercida por la motivación fonológica de las letras. Participaron 48 niños prealfabéticos entre 5 y 6 años de edad, que asistieran regularmente a escuelas públicas para la primera infancia en la región central de San Pablo, Brasil. Organizamos a los niños en 3 grupos según el nivel de escritura (en parámetros de Ferreiro y Teberosky, 1979). En entrevistas individuales, los niños realizaron tres tareas: identificar letras fuera de contexto, escribir palabras y completar palabras sin la letra inicial. Los resultados indican que el efecto de la motivación fonológica vinculada al nombre de la letra difiere entre las diferentes tareas, variando también según el nivel de escritura de los niños. Realizamos análisis multivariados para determinar: si identificar letras influye en la tarea de escribir y completar palabras; la influencia del nivel de escritural para identificar, escribir y completar palabras; y la influencia de la motivación fonológica en el rendimiento de los niños. Los resultados indican que las posibilidades del niño de usar e integrar información para escribir y completar palabras no dependen del conocimiento del nombre de la letra sino de los niveles de conceptualización que tienen respecto del funcionamiento del sistema de escritura.

Palabras clave: Emotivación fonológica; identificación de letra; nivel de escritura.

\section{THE USEFULNESS OF KNOWING THE NAME OF THE LETTERS FOR THE ACQUISITION OF THE WRITING SYSTEM}

Cognitive psychology research indicates that the knowledge of the name of the letters is essential to learn to read and write. However, psychogenetic research shows that the use of this information (name of the letter) is conditioned by the way in which the child understands the functioning of the writing system. The objective of this work was to investigate the relationship between identifying letters by their respective names and the possibility of using them in a pertinent way in writing and completing words contexts, observing the influence exerted by the phonological motivation of the letters. Forty-eight pre-alphabetical children between 5 and 6 years old, who regularly attended public schools for early childhood in the central region of São Paulo, Brazil, participated. We organized the children in 3 groups according to the writing level (in Ferreiro \& Teberosky, 1979 terms). Through individual interviews, the children performed three tasks: identifying letters out of context, writing words and completing words without the initial letter. The results indicate that the effect of the phonological motivation linked to the letter's name differs between the different tasks, also varying according to the children's writing level. We carried out multivariate analyzes to determine: if identifying letters influences the task of writing and completing words; the influence of

\section{plupais}


the writing level to identify, write and complete words; and the influence of phonological motivation on children's performance. The results indicate that the child's capacity of using and integrating information to write and complete words do not depend on the knowledge of the letter's name but on the levels of conceptualization that they have regarding the functioning of the writing system.

Keywords: phonological motivation of the letters; identifying the letters names; written level.

\section{A UTILIDADE DE CONHECER O NOME DAS LETRAS PARAA AQUISIÇÃO DO SISTEMA DE ESCRITA}

Pesquisas de natureza cognitivista apontam como fundamental o conhecimento do nome da letra para a aprendizagem da leitura e da escrita, considerando-o um pré-requisito no processo de alfabetização. No entanto, pesquisas de natureza psicogenética marcam que a utilização da informação (nome da letra) está condicionada pela maneira com que a criança entende o funcionamento do sistema de escrita. Nesse sentido, o objetivo desse trabalho foi investigar a relação entre identificar as letras por seus respectivos nomes e a possibilidade de utilizá-las pertinentemente em contextos de escrita e de completar palavras, observando a influência exercida pela motivação fonológica das letras. Solicitamos a participação de 48 crianças pré-alfabéticas, no período fonetizante, entre 5 e 6 anos, que frequentavam a última etapa da educação infantil, em unidades escolares públicas da região central de São Paulo. Organizamos as crianças em 3 grupos de 16 integrantes, segundo o nível de escrita (silábico sem valor sonoro convencional, com valor sonoro convencional, e silábico-alfabético). Em entrevistas individuais, as crianças realizaram três tarefas: identificação de letras fora de contexto, escrita de palavras e completar palavras desprovidas da letra inicial. Os resultados indicam que o efeito da motivação fonológica ligada ao nome da letra difere entre as diferentes tarefas, ou seja, pode influenciar positivamente em algumas e negativamente em outras, variando também segundo o nível conceitual de cada criança. Realizamos análise multivariada para determinar: se identificar letras influencia na tarefa de escrita e de completar palavras; a influência do nível conceitual para identificar, escrever e completar palavras; e a influência da motivação fonológica no desempenho infantil. Os resultados indicam que as possibilidades da criança para utilizar e integrar informações para escrever e completar palavras não dependem do conhecimento do nome da letra.

Palavras-chave: motivação fonológica; identificação de letras; nível de escrita.

\section{plurais}

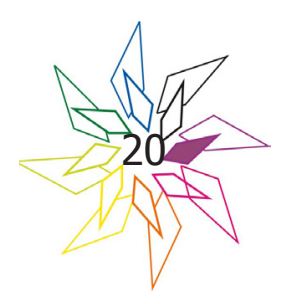




\section{LA UTILIDAD DE CONOCER EL NOMBRE DE LAS LETRAS PARA ADQUIRIR EL SISTEMA DE ESCRITURA}

\section{Introducción}

Si bien es cierto que en una cultura letrada a muy temprana edad los niños comienzan a tener contacto con la lengua escrita, se espera que, junto con el ingreso a la escuela, comiencen a aprender formalmente algunos aspectos relacionados con el sistema de escritura. Es así que se enseña el abecedario que incluye el nombre de las letras. Pese a este tipo de práctica tan arraigada en la tradición escolar, no es del todo clara la utilidad que esta información, el nombre de las letras, puede tener en el proceso de alfabetización, de ahí que nos ocupemos de este asunto en el presente estudio.

Cabe señalar, que esta misma inquietud se ha compartido por diferentes tradiciones psicolingüísticas, lo que nos dará ocasión para discutir el peso que puede tener la información en sí misma cuando se prescinde de considerar las conceptualizaciones de los niños sobre el sistema de escritura. Desde la perspectiva psicogenética, que es la que sostenemos, sabemos que los niños construyen sistemas pre-convencionales de interpretación sobre el sistema de escritura. Estas construcciones, que se dan por la interacción entre los niños y la lengua escrita, lo que influye en el sentido que dan a la información que reciben y, al mismo tiempo los lleva a realizar consideraciones progresivamente más completas de los elementos y la lógica misma del sistema de escritura. De ahí que para nuestro estudio consideremos las respuestas escritas pre-convencionales de los niños como foco central de análisis. Para ello, reconocemos especialmente pertinente la descripción en los momentos de la adquisición del sistema de escritura observado por Ferreiro y Teberosky (1979).

Es importante decir que con este estudio intentamos también continuar nuestras exploraciones sobre el peso de los rasgos fonológicos en la toma de consciencia que realizan los niños sobre el alor sonoro convencional de ciertas letras sobre otras (Fernández y Alvarado, 2015; Alvarado, 1997): la motivación fonológica del nombre de las letras (más y menos motivadas²).

2 En portugués, lengua en que se llevó el estudio, al igual que en el español, existen denominaciones para las letras más y menos motivadas fonológicamente. Una denominación motivada se da cuando el nombre de la letra comienza con el fonema que representa. Por ejemplo "TE" el nombre de la grafía " $T$ " es moti-

\section{plupais}


A diferencia de una postura psicogenética, desde una perspectiva cognitivista del aprendizaje el acento se ha puesto en la información (provista a los sujetos) más que en lo que los mismos sujetos pueden hacer cuando interactúan con un saber cultural. En este sentido, establecer qué tipo de tarea es la que mejor predice el desempeño lector o el éxito en la tarea de alfabetización, ha sido una preocupación constante. Así, el artículo reciente de Treiman et al. (2019) muestra que el predictor más confiable, respecto del éxito lector, de un conjunto de niños norteamericanos, recién egresados de preescolar, fue su capacidad para deletrear palabras de uso frecuente, sobre otras tareas entre las que se encontraban las propias de conciencia fonológica o la de identificación de palabras escritas (sight word reading).

\section{Antecedentes teóricos}

Desde una perspectiva psicogenética, encontramos que la información que poseen los niños respecto del nombre de las letras no necesariamente condiciona su actuación al momento de escribir palabras determinadas. En el trabajo de Alvarado (1997) se reportó que los niños prealfabetizados podían muchas veces identificar de manera correcta las letras que se les presentaban de manera descontextualizada. Sin embargo, esta posibilidad no estuvo relacionada con la escritura convencional o parcialmente convencional (como en el caso de niños con escrituras silábicas) de una serie de palabras con estructura silábica regular y de alta frecuencia. También observó que los niños nombraban las letras de diferentes maneras, variaban según el nivel de escritura: por su nombre o fonema (niños con escritura alfabética o silábica-alfabética) y de manera silábica o haciendo alusión a una palabra cuya sílaba inicial la incluyera; por ejemplo "la ma" o "la de Mariana" (niños con escrituras silábicas que pudieran o no representar pertinentemente estos segmentos en una palabra dada).

Por su parte, Cano y Vernon (2008) estudiaron qué información sobre las letras (conocer el nombre de la letra, identificarla por el fonema que representa o por un segmento silábico o de inicio de palabra) les resulta más útil a los niños prealfabetizados para escribir o completar palabras. En este trabajo se privilegió el análisis de las consonantes cuyos contextos silábicos coincidían con el nombre de la letra (motivación fonológica).

vado, pero no así "ESE" o "ERRE".

\section{plupais}

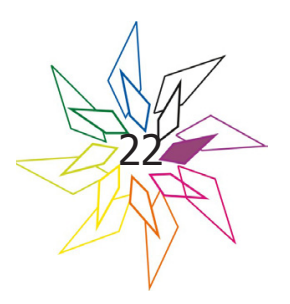


Cano y Vernon (2008) observaron que una variable importante fue el contexto silábico sobre el que los niños completaban o escribían palabras. Fue más frecuente usar pertinentemente las consonantes cuando: i) estaban acompañadas por vocales abiertas; ii) en las sílabas iniciales que formaban denominaciones motivadas de las letras consonantes ("TE", "PE", "BE"...); y iii) se involucraban segmentos con consonantes con rasgo más continuo.

Así mismo, en este trabajo se insiste en que cuando los niños tienen referentes específicos de uso de una letra (por ejemplo, en los nombres propios) esta información les resulta útil al momento, tanto de identificar letras fuera de contexto, como al usarlas para resolver las tareas de escritura o completamiento de palabras. Esta conclusión coincide con lo descrito por Ferreiro y Teberosky (1979) y Ferreiro, Gómez Palacios et al. (1982) cuando las autoras marcan la importancia nombre propio en el momento de la alfabetización inicial, como un prototipo de escritura que proporciona información sobre la organización del sistema de escritura, sus letras y combinaciones.

Por su parte los trabajos de Vernon $(1997,2005)$ señalan que las posibilidades de los niños hispanohablantes prealfabetizados, para analizar el lenguaje oral, son inferiores a las que los mismos niños presentan en presencia de las formas escritas de las palabras que se les solicita analizar fonológicamente. Así mismo, que las posibilidades de análisis fonológico siguen un orden que está estrechamente vinculado con las conceptualizaciones progresivas sobre el sistema de escritura descritas por Ferreiro y Teberosky (1979). Adicionalmente, Vernon $(1997,2005)$ señala que, dado que la escritura es un sistema de representación de una lengua, la estructura de cada lengua impondrá limitaciones a los análisis que se pueden realizar, lo que alerta respecto lo riesgoso de extender conclusiones sobre el desempeño de los niños alrededor de tareas de conciencia fonológica u otras relacionadas con la identificación o producción de palabras escritas, en contextos lingüísticos diferentes ya que la estructura de una lengua, sus especificidades fonológicas y ortográficas, influye el proceso cognitivo involucrado en la alfabetización específica en una lengua determinada.

En el trabajo de Fernández y Alvarado (2015) solicitaron a 90 niños mexicanos prealfabetizados que: i) escribieran cinco diferentes sustantivos en español para identificar sus concepciones sobre el sistema de escritura (en términos de Ferreiro y Teberosky, 1979); ii) identificaran doce diferentes palabras escritas en español que se les presentaron en una pantalla (seis de las cuales comenzaban con fonemas más continuos y el resto con menos continuos); iii) identificaran las letras del abecedario que se les presentaban en orden aleatorio.

\section{plupais}


Los resultados de este estudio muestran que independientemente del conocimiento que pudieran demostrar los niños sobre las letras del abecedario (conocer o no su denominación o el fonema que representan) el rasgo de continuidad, presente al inicio de las palabras, fue relevante en la identificación de palabras escritas, sobre todo a partir de que los niños presentaban escrituras silábicas. Cuando podían incorporar el valor de las letras pertinentemente en sus escrituras (aunque haciendo todavía representaciones silábicas) la posibilidad de identificar palabras con rasgos menos continuos se incrementó, de manera que los niños con escrituras silábico-alfabéticas pudieron reconocer la forma escrita de la mayoría de las palabras.

Estos datos son relevantes para el presente estudio dado que las letras que se emplean en español para representar los fonemas menos continuos coinciden en poseer denominaciones más motivadas ("P", “T”, “C", "B”, "D”). Los hallazgos de este estudio difieren, como lo veremos más adelante, en las conclusiones que los trabajos cognitivistas han planteado.

Desde una perspectiva cognitivista del aprendizaje, encontramos también estudios importantes sobre la utilidad de conocer el nombre de la letra para que los niños puedan llegar a leer palabras de uso frecuente. La explicación que subyace a este planteamiento marca que el conocimiento del nombre de las letras desencadena en los niños pequeños el establecimiento de relaciones fono-gráficas, ya que el nombre de la letra contiene el sonido que normalmente se representa en la escritura de palabras (Ehri,1986).

Por su parte, Treiman y Rodríguez (1999) y Pollo et al. (2005) defienden que las letras con motivación fonológica son frecuentes en muchas lenguas y, al hacer coincidir el fonema inicial del nombre de la letra con el fonema que en efecto representa, esta información favorece que los niños puedan establecer relaciones grafo-fonéticas más fácilmente. Pese a las similitudes que encuentran respecto a la denominación del nombre de las letras entre las diferentes lenguas, asumen también algunas diferencias, de ahí que encuentre mayor utilidad de conocer el nombre de las letras consonantes para los niños anglo parlantes, que el de las letras vocales. Este fenómeno se presenta a la inversa en niños hablantes de portugués (Pollo et al., 2005). Es importante destacar que la denominación de las vocales no es equivalente a la de las consonantes en portugués que en inglés. Mientras que en inglés la denominación de una vocal no necesariamente coincide con su

\footnotetext{
plurais

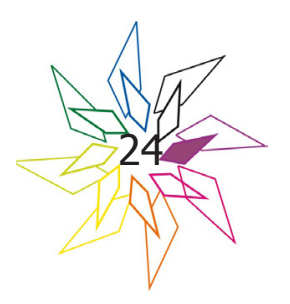


realización fonológica en una palabra escrita ${ }^{3}$, en portugués (como en español) el nombre de la letra coincide casi siempre con la realización fonológica en una palabra.

En un estudio posterior, Treiman y Bowman (2008) verificaron si los niños que no podían todavía leer y escribir, podía realizar escrituras e identificación de palabras de manera logográfica o, bien, si tenían ya algún tipo de conocimiento fonográfico. Para ello evaluaron 42 niños en edad preescolar, hablantes nativos de inglés, a quienes solicitaron que escribieran y leyeran sílabas o pseudopalabras que involucraban cuatro grupos de juegos de letras. Uno compuesto por vocales y consonantes motivadas, otro por consonantes y vocales motivadas, otro por vocales y consonantes aleatorias, y el cuarto por consonantes y vocales aleatorias. Vale la pena señalar que las letras presentadas no formaron palabras escritas en sentido estricto, sin embargo, la composición gráfica podría sugerir una palabra si se consideraba la denominación de alguna de las letras presentadas. Por ejemplo, para la palabra APE (mono, en inglés), se presentaron las letras “AP”. En el grupo de palabras arbitrarias, se presentaron letras sin relación de ningún tipo con la palabra pronunciada.

La idea era que, si la lectura y la escritura de los niños fueran logográficas, los resultados presentados para los diferentes grupos de palabras serían equivalentes. Sin embargo, los niños se desempeñaron mejor en condiciones motivadas fonéticamente que en condiciones arbitrarias. Los autores concluyeron que incluso con habilidades limitadas de lectura y escritura, los niños que tienen conocimiento del nombre de las letras no pueden limitarse a interpretaciones basadas en el enfoque logográfico, porque en su desempeño habían demostrado conocimiento, que de alguna manera habían conseguido, sobre el nombre de las letras (Treiman e Bowman, 2008).

Aunque el rendimiento fue equivalente entre vocales motivadas y consonantes, los autores observaron que la posición que ocupaba el fonema en la palabra no lo era y concluyeron que las letras motivadas ubicadas en la posición inicial demostraron ser más útiles para los niños que aquellas en posición final. También observaron un mejor desempeño de los niños con letras motivadas en las tareas de escritura que en la lectura.

Cardoso-Martins y Batista (2005) encontraron resultados similares para el portugués. En sus pruebas, los niños escribieron pares de palabras, una comenzaba con una sílaba motivada fonoló-

3 Por ejemplo, la letra "A", con denominación /ei/ sirve para escribir "Apple" donde "A" equivale a /a/, pero también podría representar al menos tres fonemas más dependiendo del contexto de conjunto (cluster) en el que se encuentre $(/ \varepsilon \mathrm{jj} /, / \mathfrak{m} /, / \mathbf{o} /)$.

\section{plurais}


gicamente (como, por ejemplo, "TELÉFONO”) y su respectivo par comenzando con sílabas sin motivación fonológica. Por ejemplo, "TORTUGA". Con este estudio llegaron a la conclusión de que "(...) los niños de habla portuguesa usan su conocimiento del nombre de las letras para conectar la escritura con el habla, al menos cuando se puede detectar el nombre completo de la letra en la pronunciación de la palabra" (Cardoso-Martins y Batista, 2005, p. 334).

Aunque autores como Treiman (1994, 1998); Treiman et al. (2001); Read y Treiman (2013); Pollo et al. (2015) han insistido en que el conocimiento de las letras podría considerarse como un elemento fundamental para comenzar el proceso de alfabetización, en sus trabajos de investigación, incluso si se lleva a cabo con niños que hablan portugués brasileño, encontramos varios aspectos metodológicos cuestionables: i) no se explica, por ejemplo, qué letras reconocen los niños y de qué manera las nombran (hay más de una forma posible de nombrar en portugués a algunas letras); ii) cuando se realizan tareas de escritura, el dictado de las palabras se realiza colectivamente, sin controlar las justificaciones que pudieran hacer los niños mientras escriben o al final de su escritura; iii) la elección de las palabras a escribir por los niños no es considerada, ni desde el punto de vista de las características del lenguaje (tipo de silabas involucradas o longitud), ni desde el punto de vista de los sujetos y sus posibilidades de conceptualización sobre el sistema de escritura, proponiendo palabras que, desde la perspectiva que desean refutar, generaría importantes conflictos cognitivos que se reflejarían en la producción (por ejemplo, cuando solicitan la escritura de monosílabos).

Otro aspecto muy discutible en los estudios presentados es que, aunque sostienen que saber el nombre de la letra es la primera entrada al sonido que representará la letra y que este conocimiento es una condición para que los niños se alfabeticen, no justifican el hecho de que muchos niños demuestran conocer las letras por sus nombres y los sonidos respectivos, pero no leen ni escriben de manera convencional.

Con estos cuestionamientos abordamos el presente estudio, sabemos que en diferentes momentos conceptuales sobre la lengua escrita, los niños enfrentan el problema de escribir, leer, identificar o completar palabras escritas de manera diferente. Nuestra hipótesis ha sido que la información que tienen sobre el sistema de escritura (nombre y sonido de la letra) se utilizará de acuerdo con las posibilidades de análisis. Por lo tanto, cuanto más avanzado sea el nivel conceptual del niño, más beneficio puede obtener de la información sobre el nombre de las letras. De tal suerte que esperamos que a pesar de que haya niños que conozcan el nombre de las letras, esta

\section{plurais}

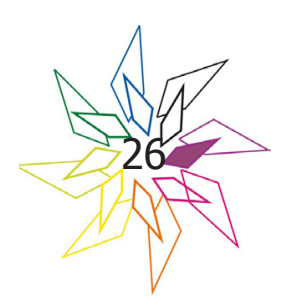


información no será útil en caso de que sus concepciones sobre el sistema de escritura sean sin uso pertinente de letras (de acuerdo con su valor sonoro, al menos para representar sílabas). La utilidad del conocimiento del nombre de las letras será mayor en tanto las conceptualizaciones sean más convencionales (con empleo de algunas letras para representar con pertinencia segmentos silábicos).

Con este estudio quisimos responder a las siguientes preguntas: ¿saber los nombres de las letras puede facilitar que los niños usen estas letras para escribir palabras?; ¿conocer el nombre de las letras les facilita la identificación de la letra inicial de una palabra determinada?; ¿el momento conceptual (nivel de escritura) condiciona la utilidad de conocer el nombre de la letra al escribir o completar palabras?

\section{Método}

Para responder a las preguntas de nuestro estudio, entrevistamos a 48 niños que asistían al último grado de Educación Infantil en escuelas públicas de la región central de la ciudad de San Pablo, Brasil, con esto nos aseguramos de que el origen social de los niños fuera equivalente. La edad promedio de los participantes fue de 5;06 años en el momento de la recopilación de datos y la muestra estuvo formada por niños y niñas de 4 escuelas diferentes, debido a la dificultad de componer 3 grupos de 16 niños por cada nivel de escritura: con escrituras silábicas sin uso pertinente de letras de acuerdo con su valor sonoro (en lo sucesivo, escrituras silábicas sin pertinencia sonora o SSPS); con escrituras silábicas con uso pertinente de algunas letras (en lo sucesivo, escrituras silábicas con pertinencia sonora o SCPS); o con escrituras silábico-alfabéticas con uso pertinente de algunas letras (en lo sucesivo, escrituras silábico-alfabéticas).

Todos los niños que participaron en este estudio fueron entrevistados individualmente en sesiones de 40 minutos, en los que les pedimos que realizaran tres tareas:

1) Tarea de escritura de sustantivos para conocer el nivel de conceptualización del lenguaje escrito en el que se encontraban, de acuerdo con los parámetros descritos por Ferreiro y Teberosky (1979) y observar las decisiones gráficas que tomaban para escribir las palabras dictadas. Como lo veremos más adelante, seleccionamos palabras que nos permitieran evaluar la escritura que realizaban para segmentos silábicos iniciales más o menos motivados, con respecto a la letra inicial.

\section{plurais}


El establecimiento del nivel de escritura que presentaron los niños lo realizamos bajo criterios estadísticos equivalentes a los establecidos por Fernández y Alvarado (2015): consideramos que un niño tenía escritura silábica cuando representaba el 75\% de los segmentos silábicos de las palabras dictadas (al menos 26 sílabas de un total de 36), empleando una grafía que justificaba de manera silábica; consideramos que se trataba de una escritura silábica con uso pertinente de letras (SCPS) cuando al menos el 75\% de los segmentos silábicos representados, en el conjunto total de las palabras dictadas, presentaba letras pertinentes; consideramos que se trataba de una escritura silábica sin uso pertinente de letras (SSPS) cuando los segmentos representados no rebasaban el 75\% de uso de letras pertinentes; y identificamos escrituras silábicas -alfabéticas cuando la representación del al menos el 50\% de los segmentos silábicos del total de las palabras dictadas (al menos 18 segmentos silábicos de un total de 36) presentaban representaciones gráficas intra-silábicas y el resto eran silábicas.

Para la tarea de escritura dictamos a los niños doce sustantivos trisilábicos con estructura silábica regular en portugués (CV-CV) que fueron extraídos del diccionario "Mini Aurélio escolar". Esta decisión la tomamos al considerar que el portugués es una lengua predominantemente trisílaba con estructura silábica canónica CV (Marques, 2008). Adicionalmente, los trisílabos evitarían interferencias en las reflexiones de los niños de acuerdo con el establecimiento del criterio de cantidad expuesto por Ferreiro y Teberosky (1979), que les complicaría la realización de escrituras de mono y bisílabos.

Otro criterio que también se consideró al elegir las palabras fue la variabilidad entre las sílabas que las componían, ya que otra hipótesis secundaria para los niños es que para que algo se escriba, debe haber variación entre los caracteres de una cadena gráfica. Por lo tanto, todas las palabras estarían compuestas de diferentes sílabas contiguas.

No sólo consideramos en general palabras escritas con letras iniciales más y menos motivadas, sino que elegimos los inicios de estas palabras en función de la frecuencia de uso en el portugués brasileño, siguiendo lo descrito por Quaresma y Pinho (2007). Adicionalmente, consideramos la transparencia ortográfica de las combinaciones silábicas de las palabras (sobre todo de la primera sílaba).

\section{plurais}

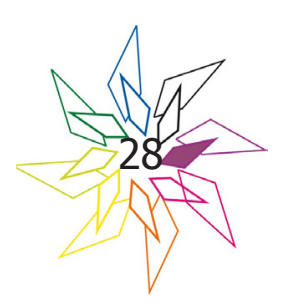


Los sustantivos empleados para esta tarea fueron organizados en tres grupos de cuatro palabras, cuyas sílabas iniciales siguieron los siguientes criterios:

a) Palabras con sílabas iniciales motivadas: palabras cuyas primeras sílabas coinciden completamente con el nombre de la consonante con el contexto silábico, es decir, que están motivadas fonológicamente. Por ejemplo, en la palabra "TEMIDA" la letra "T" se llama "Tê" y coincide con el sonido formado por la sílaba $\mathrm{T}+\mathrm{E}$.

b) Palabras con sílabas contrastantes iniciales: palabras en las que las primeras sílabas estaban formadas por las mismas consonantes motivadas utilizadas en el grupo de palabras anterior, pero en un contexto silábico distinto, de modo que la sílaba no estaba motivada y servía para contrastar con el primer grupo de palabras, por ejemplo, en la palabra "TOMADA".

c) Palabras con sílabas iniciales no motivadas: palabras en las cuales las primeras sílabas estaban compuestas de letras que, independientemente del núcleo silábico, no presentan motivación fonológica. Por ejemplo, "RAPINA".

Cada niño escribió una a una las palabras y la entrevistadora les pidió que justificaran su producción, de manera que pudiera verificar el criterio de escritura de cada niño. Por su parte, el niño podía hacer modificaciones a partir de su justificación (borrar, agregar o sustituir elementos). Cuando esto sucedía, se les solicitaba una nueva justificación de la escritura lograda. Las palabras dictadas se presentan en la Tablal (en el paréntesis aparece la traducción de los sustantivos en español).

Tabla 1: Categorías de palabras, según la motivación fonológica de la primera sílaba

\begin{tabular}{|c|c|c|}
\hline $\begin{array}{c}\text { Palabras con sílabas iniciales } \\
\text { motivadas }\end{array}$ & $\begin{array}{c}\text { Palabras con sílabas iniciales } \\
\text { de contraste }\end{array}$ & $\begin{array}{c}\text { Palabras con sílabas ini- } \\
\text { ciales no motivadas }\end{array}$ \\
\hline Temida (temido) & Tomada (enchufe) & Rapina (presa) \\
\hline Peluda (peludo) & Paleta (paleta) & Novena (novena) \\
\hline Verina (Verina) & Víbora (víbora) & Muleta (muleta) \\
\hline Bexiga (vejiga) & Buzina (bocina) & Luneta (catalejo) \\
\hline
\end{tabular}

Fuente: Datos obtenidos en la investigación de campo y organizados por las autoras.

\section{plurais}


El dictado se inició con una palabra motivada, seguida de una palabra no motivada, seguida de una palabra del grupo de contraste. El orden de dictado fue evitando que dos palabras dictadas de manera contigua compartieran la misma inicial.

2) Tarea de identificación de letras fuera de contexto. Con esta tarea quisimos observar cómo denominaban a las letras que se les mostraban en desorden, una a una, considerando el total de las letras del abecedario. Esta tarea fue similar a la utilizada por Cano y Vernon (2008) y Fernández y Alvarado (2015).

3) Tarea de completar palabras escritas a las que les faltaba la inicial, para observar si en este contexto los niños usaban el conocimiento que tenían sobre los nombres de las letras y si la coincidencia entre el contexto silábico y el nombre de la letra, es decir, la motivación fonológica, era información relevante para realizar esta tarea.

Para esta tarea, presentamos individualmente doce palabras escritas sin la letra inicial (su lugar estaba marcado por un espacio en blanco, subrayado), para que los niños seleccionaran la inicial entre cinco opciones de letras. El entrevistador, siguiendo el protocolo de Alvarado (1997), presentó la palabra escrita, omitiendo la letra inicial, y proporcionó a los niños la emisión oral de la palabra completa, usando la consigna: "En esta palabra (refiriéndose a la escritura) quiero que diga, por ejemplo, "PATO", pero le falta la primera letra, así dice "ATO” (mencionando la palabra sin la letra inicial). ¿Cuál de estas letras (señalando las letras escritas) sirve para que diga PATO”.

Seleccionamos palabras con letras iniciales tanto motivadas como no motivadas fonológicamente. Las palabras empleadas siempre fueron bisílabas con estructura CV - de alta frecuencia en el portugués brasileño -, que además presentara variedad tanto en los núcleos silábicos como en las consonantes. Asimismo, cuidamos que las sílabas iniciales de las palabras fueran ortográficamente transparentes.

Al igual que en la tarea de escritura de sustantivos, en ésta incluimos palabras de contraste en las que el segmento silábico inicial, aunque incluía la consonante motivada, la vocal no correspondía con la denominación. En la Tabla 2 presentamos las palabras empleadas (en el paréntesis aparece la traducción de los sustantivos en español).:

\section{plurais

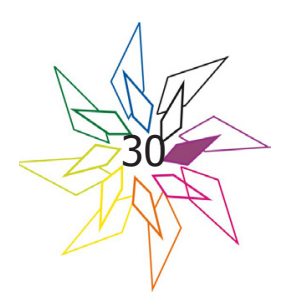


Tabla 2: Categorías de palabras, según la motivación fonológica de la primera sílaba.

\begin{tabular}{ccc}
\hline Con motivación & Contraste & Sin motivación \\
\hline Tema (tema) & Toma (toma) & Rato (ratón) \\
Pena (pluma) & Puma (puma) & Lima (lima) \\
Vejo (veo) & Vila (pueblo) & Mula (mula) \\
Beco (callejón) & Boca (boca) & Nuca (nuca) \\
\hline
\end{tabular}

Fuente: Datos obtenidos en la investigación de campo y organizados por las autoras.

Para completar cada palabra, se pusieron a disposición cinco letras para que el niño eligiera la que resultara pertinente. Entre las letras mostradas estaba la letra "meta" (ortográficamente pertinente), la vocal involucrada en la sílaba inicial, otra vocal cualquiera, una consonante fonológicamente cercana a la letra "meta", y una consonante "distante" que difiriera de la meta en más de dos rasgos fonológicos.

Cabe señalar que las letras que se mostraron a los niños se presentaban dispuestas en desorden sobre la mesa para evitar cualquier sugerencia de inicio o fin.

\section{Resultados}

Sobre la identificación de letras del abecedario, los niños participantes demostraron conocer la mayoría de las letras, siendo que $71.79 \%$ de las respuestas fueron pertinentes, es decir, decían el nombre convencional de la letra mostrada, el fonema que representa o un contexto silábico o de palabra en que efectivamente se encuentra. Por ejemplo, dijeron "la de María" o la "MA" o "la /m/", o "la eme" para la letra "M".

Encontramos que fue más sencillo para los niños identificar las vocales que las consonantes (85.41\% Vs. 69.96\% respectivamente) de respuestas pertinentes. Respecto de las consonantes el porcentaje fue de $69.96 \%$. Al realizar una prueba de Chi cuadrada encontramos una significancia estadística que muestra correlación entre el tipo de letra (consonante o vocal) e identificar la letra (respuestas pertinentes o no pertinentes) con una $\alpha=0.007$.

\section{plurais}


Respecto de la motivación de las letras consonantes, no identificamos que existiera correlación estadística entre el tipo de respuesta (pertinente o no pertinente) por el tipo de denominación de la letra (motivada o no motivada). Para las letras motivadas encontramos $68 \%$ de respuestas correctas, mientras que para las no motivadas el porcentaje fue $73 \%$.

Respecto de la identificación de letras por el nivel de escritura de los participantes, encontramos correlaciones entre ambas variables. En la Tabla 3 resumimos los puntajes por nivel de escritura.

Tabla 3. Frecuencias y porcentajes de respuestas pertinentes en la tarea de identificación de letras, por nivel de escritura

\begin{tabular}{llcc}
\hline \multicolumn{1}{c}{ Nivel de escritura } & & Vocal & Consonante \\
\hline \multirow{2}{*}{ SSPS } & Frecuencia & 54.00 & 140.00 \\
& Porcentaje & 67.50 & 46.10 \\
\multirow{2}{*}{ SCPS } & Frecuencia & 72.00 & 209.00 \\
& Porcentaje & 90.00 & 68.80 \\
\multirow{2}{*}{ SA } & Frecuencia & 80.00 & 291.00 \\
& Porcentaje & 100.00 & 95.70 \\
\hline \multirow{2}{*}{ Total de respuestas } & Frecuencia & 206 & 640 \\
& Porcentaje & 85.80 & 70.20 \\
\hline
\end{tabular}

Fuente: Datos obtenidos en la investigación de campo y organizados por las autoras.

Como puede apreciarse en la tabla anterior, las respuestas correctas, tanto para vocales como para consonantes se incrementaron en relación directa con el nivel de escritura. Adicionalmente, calculamos el Coeficiente de Correlación de Spearman y encontramos una correlación significativa, entre las identificaciones de vocales y el nivel de escritura $s=0.643$ y entre identificación de consonantes y nivel de escritura $s=0.679$ con $p=0.01$ (bilateral). De acuerdo con estos resultados, podemos afirmar que existe correlación entre las posibilidades de identificar letras del abecedario y el nivel de escritura de los niños participantes.

Sobre la tarea de escritura de sustantivos diremos que además de permitirnos identificar el nivel de conceptualización sobre el sistema de los niños de nuestra muestra, nos demandó el análisis de la escritura del primer segmento silábico de las palabras dictadas, para poder obser- 
var si los niños en este contexto empleaban letras de acuerdo con su valor fonológico, aunque lo hicieran para representar una sílaba. En este sentido encontramos cuatro diferentes tipos de respuestas: (1) representar la sílaba inicial empleando tanto la consonante como la vocal pertinente; (2) representar la sílaba inicial empleando solo la consonante pertinente; (3) representar la sílaba inicial empleando solo la vocal pertinente; y (4) representar la sílaba inicial empleando una letra (consonante o vocal) no pertinente.

La Tabla 4 resume el porcentaje y frecuencia de respuestas para la escritura de la sílaba inicial de los substantivos dictados.

Tabla 4. Frecuencia y porcentaje de respuestas en la tarea de escritura por tipo de sílaba inicial $(N=192)$

\begin{tabular}{llccccc}
\hline $\begin{array}{c}\text { Tipo de sílaba } \\
\text { inicial }\end{array}$ & & $\begin{array}{c}\text { CV } \\
\text { Pertinentes }\end{array}$ & $\begin{array}{c}\text { Vocal } \\
\text { pertinente }\end{array}$ & $\begin{array}{c}\text { Consonante } \\
\text { Pertinente }\end{array}$ & $\begin{array}{c}\text { No } \\
\text { pertinente }\end{array}$ & $\begin{array}{c}\text { Total por } \\
\text { línea }\end{array}$ \\
\hline \multirow{2}{*}{ Motivadas } & Frecuencia & 13 & 15 & 109 & 55 & 192 \\
& Porcentaje & 6.77 & 7.81 & 56.77 & 28.65 & 100 \\
\multirow{2}{*}{ Contraste } & Frecuencia & 52 & 55 & 31 & 54 & 192 \\
& Porcentaje & 27.08 & 28.65 & 16.15 & 28.13 & 100 \\
No motivadas & Frecuencia & 47 & 72 & 17 & 56 & 192 \\
& Porcentaje & 24.48 & 37.50 & 8.85 & 29.17 & 100 \\
\hline \multirow{2}{*}{$\begin{array}{l}\text { Total de respu- } \\
\text { estas }\end{array}$} & Frecuencia & 112 & 142 & 157 & 165 & 576 \\
& Porcentaje & 19.44 & 24.65 & 27.26 & 28.65 & 100 \\
\hline
\end{tabular}

Fuente: Datos obtenidos en la investigación de campo y organizados por las autoras.

Si consideramos que las tres primeras categorías de respuestas encierran representaciones pertinentes (aunque sean silábicas) en tanto que los niños emplean alguna letra de acuerdo con el valor sonoro que representa, las respuestas pertinentes de escritura fueron más que las no pertinentes $(71.35 \%$ fueron pertinentes).

Dentro de la misma Tabla 4, resaltan las respuestas escritas para las sílabas motivadas. En ellas observamos que más de la mitad de los niños (56.77\%) representaron la sílaba inicial empleando la consonante pertinente, pero prescindieron de la vocal involucrada. Es decir, fue más frecuente utilizar la consonante pertinente para representar un segmento silábico "motivado" que 
otro segmento "no motivado" o "contrastante". Sin embargo, las escrituras más convencionales (en las que los niños usaron tanto la consonante como la vocal para representar el segmento silábico inicial) ocurrieron con mayor frecuencia en sílabas "contrastantes" o "no motivadas": la presencia de la motivación de la sílaba estaba relacionada con la escritura menos avanzada (silábica, que solo usaba una consonante).

Al realizar pruebas estadísticas, no encontramos diferencias significativas entre los tres tipos de sílabas representadas.

Analizamos también la escritura de las sílabas iniciales de las palabras considerando el nivel de escritura de los niños. Encontramos nuevamente que a medida que los niveles de escritura eran más avanzados, se incrementaron las respuestas pertinentes. La Figura 1. representa los porcentajes de respuestas obtenidas (pertinentes y no pertinentes) por nivel de escritura.

Figura 1. Tipo de respuesta por nivel de escritura

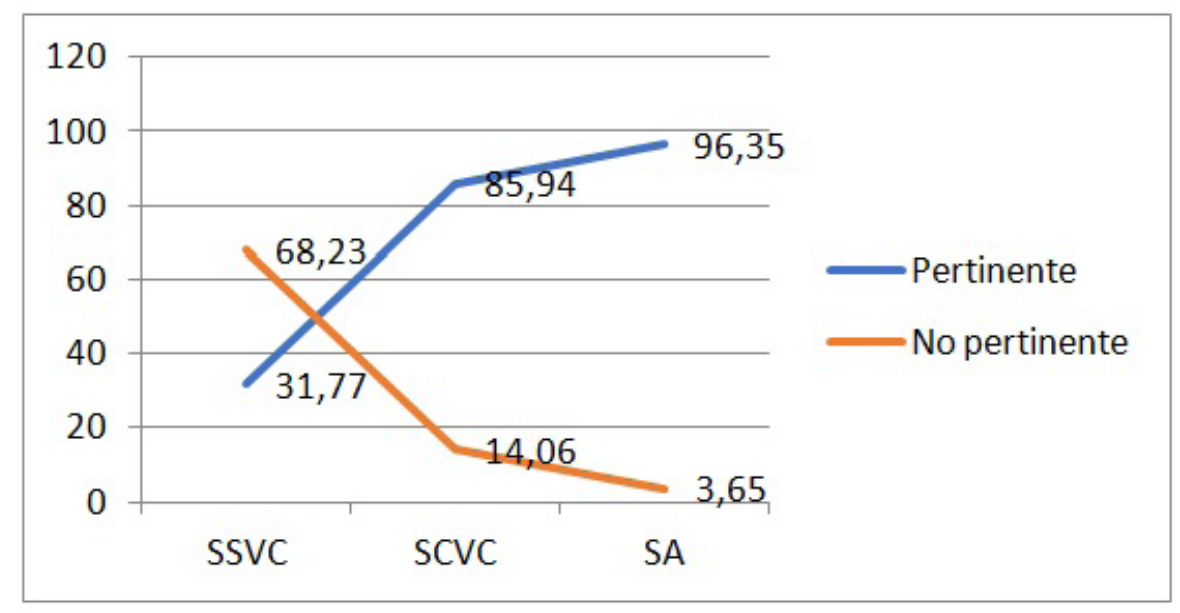

Fuente: Datos obtenidos en la investigación de campo y organizados por las autoras.

Cabe señalar la distancia tan grande entre las respuestas pertinentes de los niños con escrituras SSPS en comparación con los SCPS, y cómo la diferencia entre los niños con escrituras SCPS con

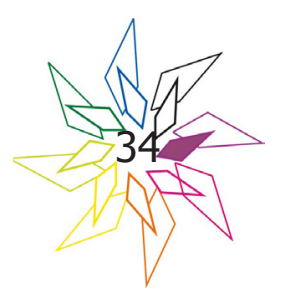


respecto a los niños con escrituras SA decrece. Estos datos nos sugieren que la diferencia podría estar asociada con la posibilidad de realizar escrituras con incorporación de letras pertinentes. Bajo esta sospecha, reanalizamos las respuestas agrupando en dos a los niños: aquellos con escrituras con incorporación de letras con pertinencia (SCPS y S-A) y aquellos con escrituras sin incorporación de letras con pertinencia (SSPS). Desde esta perspectiva obtuvimos una Chi cuadrada con $\alpha=0.000$, lo que señala correlación entre presentar respuestas pertinentes y haber incorporado letras con pertinencia en la escritura de los niños.

Realizamos un tercer análisis sobre la escritura de las palabras (en tanto a la representación de la sílaba inicial), el tipo de sílaba involucrada y el nivel de escritura. El resumen de esta información la presentamos en la Tabla 5 en donde puede apreciarse tanto la similitud entre el porcentaje total de respuestas pertinentes para los diferentes tipos de sílabas, como la progresión directa entre el nivel de escritura y el porcentaje de respuestas pertinentes.

Tabla 5. Frecuencia y porcentajes de respuestas pertinentes por nivel de escritura y tipo de sílabas $(\mathrm{N}=411)$

\begin{tabular}{|c|c|c|c|c|c|}
\hline $\begin{array}{c}\text { Nivel } \\
\text { de escritura }\end{array}$ & & Motivada & Contraste & No motivada & Total por línea \\
\hline \multirow{3}{*}{ SSPS } & Frecuencia & 22 & 21 & 18 & 61 \\
\hline & $\begin{array}{l}\% \text { nivel esc } \\
(\mathrm{n}=192)\end{array}$ & 36.1 & 34.4 & 29.5 & 100.0 \\
\hline & $\%$ total $(n-=411)$ & 5.35 & 5.11 & 4.38 & 14.84 \\
\hline \multirow{3}{*}{ SCPS } & Frecuencia & 55 & 56 & 54 & 165 \\
\hline & $\begin{array}{l}\% \text { nivel esc } \\
(\mathrm{n}=192)\end{array}$ & 33.3 & 33.9 & 32.7 & 100.0 \\
\hline & $\%$ total $(n-=411)$ & 13.38 & 13.63 & 13.14 & 40.15 \\
\hline \multirow{3}{*}{ SA } & Frecuencia & 60 & 61 & 64 & 185 \\
\hline & $\begin{array}{l}\% \text { nivel esc } \\
(\mathrm{n}=192)\end{array}$ & 32.4 & 33.0 & 34.6 & 100.0 \\
\hline & $\%$ total $(n-=411)$ & 14.60 & 14.84 & 15.57 & 45.01 \\
\hline \multirow{2}{*}{$\begin{array}{l}\text { Total de respu- } \\
\text { estas }\end{array}$} & Frecuencia & 137 & 138 & 136 & 411 \\
\hline & $\%$ total & 33.33 & 33.58 & 33.09 & 100.00 \\
\hline
\end{tabular}

Fuente: Datos obtenidos en la investigación de campo y organizados por las autoras.

\section{plupais}


Dado que la escritura convencional del segmento silábico (CV) fue integrado en el análisis de respuestas pertinentes, quisimos diferenciarlo para ver su efecto sobre el nivel de escritura, sobre todo al considerar la diferencia entre hacer representaciones silábicas (con una sola grafía para el segmento completo) y representaciones realmente alfabéticas.

Al mirar los datos de esta manera, encontramos una correlación, mediante la prueba de Rho de Sperman, significativa entre el nivel de escritura y escrituras CV con inicio motivado $s=.545$; entre nivel de escritura y escrituras CV con inicio no motivado $s=.810$ y el nivel de escritura de los niños; entre nivel de escritura y escrituras CV con inicio de contraste $s=.844$; la correlación fue significativa con $p=0.01$ (bilateral)

Respecto de la tarea de completar palabras escritas que carecían de la letra inicial, resumimos las respuestas en dos: pertinente (cuando la letra seleccionada en efecto era la letra "meta") y no pertinente (cuando la letra seleccionada era diferente de la "meta"). Los resultados los representamos en la Figura 2.

Figura 2. Frecuencia de respuestas pertinentes y no pertinentes por grupo de palabras $(n=576)$

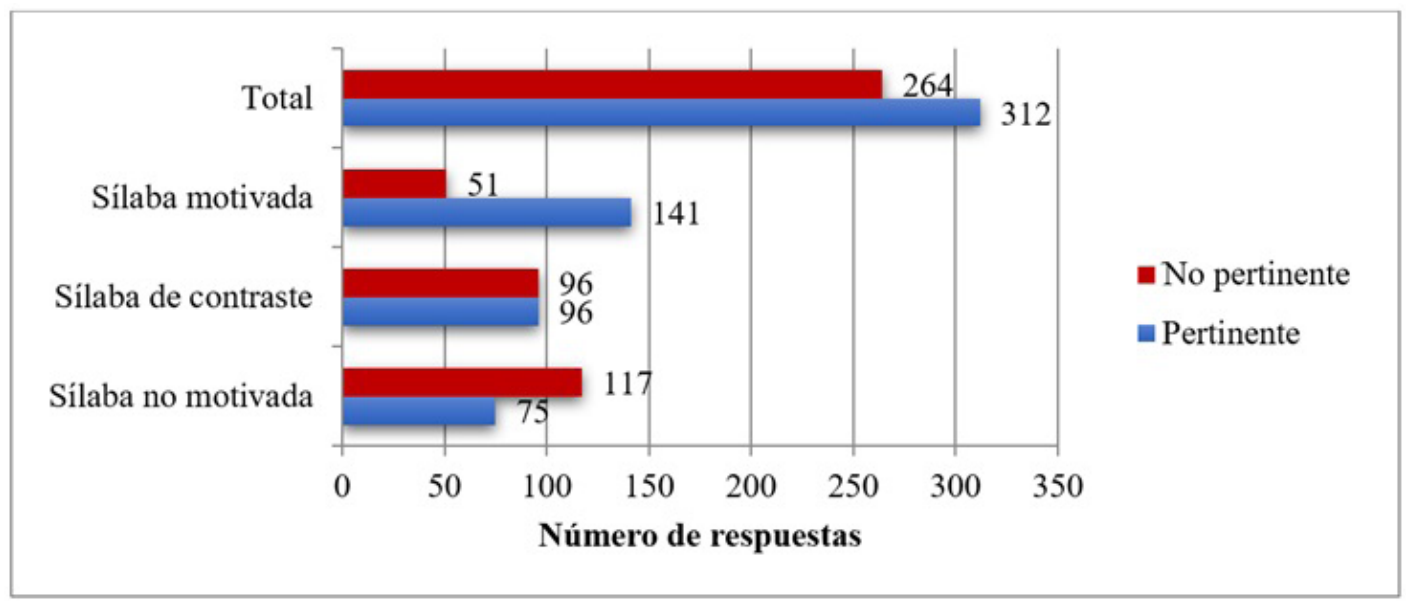

Fuente: Datos obtenidos en la investigación de campo y organizados por las autoras.

Al igual que en la tarea de escritura de sustantivos, fue más sencillo para los niños encontrar la letra inicial de palabras con inicio silábico motivado. A diferencia de los resultados de la tarea de 
escritura, en la de identificación de iniciales fue más sencillo completar los inicios de palabras con sílabas "de contraste" que con sílabas "no motivadas".

Al evaluar las respuestas convencionales de los niños por nivel de escritura, encontramos correlaciones estadísticamente significativas para todos los tipos de sílabas iniciales a través de la prueba de Spearman. Entre el nivel de escritura y completar palabras con inicial motivada $s=.5445$; entre el nivel de escritura y completar palabras de contraste $s=.635$; entre el nivel de escritura y completar palabras con inicial no motivada $s=.624$. La correlación fue significativa al nivel $\mathrm{p}=0.01$ (bilateral).

Una vez que analizamos todas las tareas del estudio, determinamos la correlación entre las posibilidades de los niños para identificar las letras del abecedario y sus posibilidades para, por una parte, escribir la sílaba inicial de los sustantivos dictados y, por la otra, encontrar las letras iniciales para completar la escritura de palabras.

Encontramos correlación estadísticamente significativa entre la identificación de letras motivadas y la escritura de segmentos silábicos iniciales coincidentes con las letras motivadas. Esto fue así, también al escribir segmentos silábicos de contraste. Por ejemplo, quién identificaba la letra "T" era más probable que pudiera escribir con la inicial correcta "TEMIDA" y "TOMADA", aunque su representación fuera silábica.

Determinamos la correlación entre variables a través de la prueba de Pearson donde obtu$\operatorname{vimos} r(48)=.745, p=0.01$ (bilateral)

Respecto de la correlación entre letras no motivadas y la escritura de un segmento silábico no motivado encontramos también una correlación estadísticamente significativa a través de la prueba de Pearson $r(48)=.618, p=0.001$ (bilateral).

Respecto de la tarea de completar con la letra inicial las palabras escritas, encontramos nuevamente correlación estadísticamente significativa, a través de la prueba de Pearson, entre quienes identificaron las letras motivadas y quienes completaron sílabas iniciales tanto motivadas como de contraste. La correlación entre identificar letras motivadas y completar iniciales motivadas de palabras escritas arrojó $r(48)=.529$; la correlación entre identificar letras motivadas y completar

\section{plurais}


iniciales de contraste en palabras escritas arrojó $r(48)=.672$. Para ambos casos la correlación fue significativa con $p=0.01$ (bilateral)

Finalmente, consideramos todas las respuestas de los niños emitidas para las tres tareas de nuestro estudio para realizar un análisis multivariado. Para ello distinguimos 8 diferentes tipos de respuestas combinadas, que presentamos a continuación en orden descendente (Tabla 6). Cabe señalar que dimos como respuesta pertinente en la tarea de escritura de sustantivos, cuando los niños emplearon la consonante inicial involucrada en la primera sílaba (independientemente de que su escritura pudiera ser silábica).

Tabla 6. Descripción de respuestas combinadas

\begin{tabular}{|c|c|c|c|c|}
\hline \multirow[b]{2}{*}{$\begin{array}{l}\text { Tipo de } \\
\text { respuesta }\end{array}$} & \multirow[b]{2}{*}{$\begin{array}{l}\text { Frecuencia de } \\
\text { respuestas } \\
\text { registradas } \\
\text { (porcentaje) }\end{array}$} & \multicolumn{3}{|c|}{ Tareas } \\
\hline & & $\begin{array}{l}\text { Identificación de } \\
\text { letras fuera de } \\
\text { contexto }\end{array}$ & $\begin{array}{c}\text { Escritura de sustantivos } \\
\text { (representación del } \\
\text { segmento silábico } \\
\text { inicial) }\end{array}$ & $\begin{array}{l}\text { Completar la letra } \\
\text { inicial faltante de } \\
\text { una palabra escrita }\end{array}$ \\
\hline $\bar{A}$ & $154(42.7)$ & $\sqrt{ }$ & $\checkmark$ & $\sqrt{ }$ \\
\hline B & $37(10.10)$ & $\checkmark$ & $x$ & $\checkmark$ \\
\hline $\mathrm{C}$ & $18(4.92)$ & $\checkmark$ & $\checkmark$ & $x$ \\
\hline D & $8(2.18)$ & $x$ & 2 & 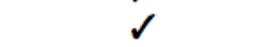 \\
\hline $\mathrm{E}$ & $67(18.30)$ & $\checkmark$ & $x$ & $x$ \\
\hline $\mathrm{F}$ & $19(5.19)$ & $x$ & $x$ & 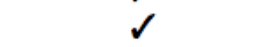 \\
\hline G & $6(1.64)$ & $x$ & $\checkmark$ & $x$ \\
\hline $\mathrm{H}$ & 57 (15.57) & $x$ & $x$ & $x$ \\
\hline
\end{tabular}

Fuente: Datos obtenidos en la investigación de campo y organizados por las autoras.

Reagrupamos las respuestas combinadas y les otorgamos un puntaje para poder analizarlas con relación al nivel de escritura: 1) al conjunto de las respuestas tipo A les dimos un puntaje de 100 por frecuencia de respuestas de este tipo; 2) al conjunto de respuestas B, C y D les dimos un puntaje de 10 por frecuencia de respuestas de este tipo; 3) al conjunto de respuestas E, F y G les dimos un punto por frecuencia de respuestas; y 4) a las respuestas $\mathrm{H}$ no les otorgamos puntos.

Bajo esta nueva óptica los resultados, por nivel de escritura los resumimos a continuación:

\section{plurais


Tabla 7. Puntajes (totales, máximos y mínimos) y porcentajes de respuestas combinadas por nivel de escritura

\begin{tabular}{ccccc}
\hline Nivel escritura & Puntaje & Porcentaje & Puntaje máx & Puntaje mín \\
\hline SSPS & 1,304 & 8.19 & 321 & 1 \\
SCPS & 4,399 & 27.62 & 611 & 7 \\
S-A & 10,226 & 64.20 & 404 & 800 \\
Total & 15,929 & 100.00 & & \\
\hline
\end{tabular}

Fuente: Datos obtenidos en la investigación de campo y organizados por las autoras.

Como se muestra en la Tabla 7, encontramos una relación directa y proporcional entre el nivel de escritura y los puntajes obtenidos en las respuestas combinadas de los participantes. En consecuencia, encontramos una correlación estadísticamente significativa, a través del establecimiento del Coeficiente de Correlación Rho de Spearman entre el nivel de escritura y los puntajes de respuestas combinadas: $s=.833, p=0.01$ (bilateral).

\section{Conclusiones}

La primera hipótesis que guió este trabajo fue que el nivel de conceptualización sobre el sistema de escritura presentado por los niños (prealfabético) condiciona sus posibilidades de identificar letras fuera del contexto de lectura, escribir y completar palabras. Es decir, cuanto mayor sea el nivel de escritura, mayores serán sus posibilidades de identificar letras, escribir y completar palabras.

Los datos recopilados en nuestro estudio en portugués, muestra que, efectivamente, cuanto mayor sea el nivel de escritura, mejor será el rendimiento en estas tareas, lo que demuestra que el nivel de escritura es una variable fundamental, porque amplía las posibilidades de los niños para usar e integrar diferentes conocimientos. En este sentido nuestros resultados corroboran los reportados con niños hispanohablantes de autores como Alvarado (1997); Cano y Vernon (2008); Ferreiro (2000, 2007); Vernon (2001, 2005); Zamudio (2008).

Mostramos además que los niños prealfabéticos también pueden identificar letras y completar la escritura de palabras (identificar la inicial) lo que coincide con lo reportado por Cano y Vernon (2008); Fernández y Alvarado (2015) entre otros.

\section{plurais}


A diferencia de lo que respalda la teoría cognitiva (Treiman y Bowman, 2008), pudimos mostrar que los niños en el proceso de alfabetización pueden escribir y completar palabras basadas en información contextual o lingüística, lo que muestra que la actuación inteligente de los niños puede verse favorecida por algunos contextos (como lo sería la escritura de sílabas motivadas o completar palabras con inicio motivado, pero los límites de sus respuestas sólo pueden explicarse al considerar las hipótesis desde las que se explican el sistema de escritura. Esto fue particularmente evidenciado en las respuestas que obtuvimos de los niños con escrituras silábicas SSPS.

La tarea de identificar letras nos permitió concluir que los niños de la muestra tienen un amplio conocimiento sobre el nombre de las letras. Sin embargo, las identificaciones no fueron equivalentes entre vocales y consonantes, siendo las primeras más frecuentemente reconocidas que las segundas.

También observamos que a medida que aumenta el nivel de conceptualización sobre el sistema de escritura, aumenta la posibilidad de los niños de reconocer el nombre de las letras presentadas, es decir, la posibilidad de identificar letras también estaba vinculada a la reflexión que el niño hacía del sistema de escritura.

Otro aspecto que también tuvo influencia fueron las características relacionadas con la motivación fonológica (coincidencia entre el nombre de la letra y el contexto silábico), ya que los datos revelan que, estadísticamente, no era equivalente identificar letras motivadas y letras no motivadas, siendo esta última identificado con mayor frecuencia (en coincidencia con el trabajo de Alvarado y Fernández, 2015).

Aunque parece intuitivamente más fácil identificar letras cuyos nombres coinciden con los fonemas respectivos que los representan, por ejemplo, la letra T se llama "te" y se pronuncia /t/, los datos recopilados y analizados en esta investigación nos permiten concluir que las letras que carecen de esta motivación fueron identificadas con mayor frecuencia. En otras palabras, la motivación fonológica no fue un factor que la influyó.

En cuanto a la tarea de escribir palabras, los niños también demostraron un gran conocimiento, ya que el $71.35 \%$ produjo escritos relevantes para representar la primera sílaba.

Mientras que los niños con escritura silábicas (SSPS y SCPS), en el grupo de palabras contrastantes y no motivadas, sistemáticamente prefirieron usar vocales para representar los diferentes segmentos silábicos, los niños con escrituras silábica-alfabéticas usaron principalmente consonantes.

\section{plurais}

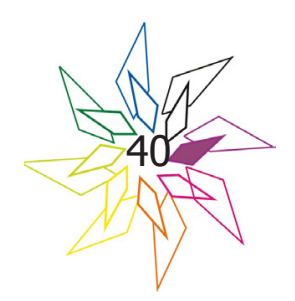


Sin embargo, en el contexto motivado, la representación mayoritaria de la sílaba, en todos los niveles conceptuales, fue por consonante. Por lo tanto, el uso de la consonante o vocal o la posibilidad de escribir la sílaba completa estuvieron directamente influenciados por la motivación fonológica de la sílaba a representar. Este fenómeno se observó tanto en la tarea de escritura como al completar palabras, pero no necesariamente impactó en la calidad de la escritura: por un lado, la sílaba motivada favorecía la selección por la consonante respectiva, por otro lado, las sílabas contrastantes y no motivadas favorecían el uso de vocales. Esta diferencia entre los tipos de respuestas no representa una diferencia cualitativa en relación con el análisis y la reflexión que el niño hace sobre el sistema de escritura, ya que el resultado obedeció a escrituras igualmente silábicas. Las respuestas de los niños con escrituras S-A apoyan también esta explicación: muchos niños con escrituras S-A que pudieron usar CV para representar sílabas de "contraste" y "no-motivadas" usaron solo una consonante en el contexto motivado, evidenciando con esto el relativo beneficio de la motivación de las letras.

En este sentido, nuestros datos apuntan, nuevamente a priorizar las conceptualizaciones de los niños sobre el sistema de escritura sobre la información que pudieran tener del nombre de las letras lo que va en contra de la idea defendida por Cardoso-Martins y Batista (2005); Pollo et al. (2005, 2015); Treiman y Bowman (2008); Treiman et al. (2013), entre otros, respecto a la utilidad que el conocimiento del nombre de la letra tiene, en sí mismo, sobre el aprendizaje de la lectura o de la escritura.

De acuerdo con los argumentos antes expuestos, podemos reinterpretar el desempeño de los niños al completar palabras escritas. Nuevamente, el nivel de conceptualización de los niños sobre el sistema de escritura fue la variable que explicó el desempeño en esta tarea en la que encontramos una correlación entre ambas variables. Así mismo, como lo explicábamos arriba, la motivación de las letras iniciales tuvo su efecto al permitir respuestas convencionales entre los niños que presentaban escrituras con uso sonoro pertinente de las letras (SCPS y S-A). De tal manera que las palabras con segmentos iniciales de contraste recibieron respuestas en las que los niños con escrituras SCPS eligieron vocales, incluso entre quienes habían identificado correctamente las iniciales en contextos motivados. Este mismo tipo de respuestas las observamos en los niños con escrituras SSPS que incluso conocían el nombre de las letras involucradas para completar palabras no motivadas.

Aunque todos los niños, en la tarea de completar, se desempeñaron mejor en el grupo de palabras motivadas, la diferencia entre los grupos de palabras de los niños S-A no fue significativa,

\section{plupais}


ya que presentaron un rendimiento equilibrado en los tres grupos de palabras. Para los niños SSPS y SCPS, el número de palabras correctas motivadas representaba casi el doble en comparación con las demás.

La segunda y tercera hipótesis de este estudio están relacionadas con la generalización de la posibilidad de utilizar una letra conocida. Propusimos (en la segunda hipótesis) que conocer el nombre de las letras puede facilitar que los niños usen esa letra de manera pertinente para escribir palabras (al menos al representar el segmento silábico inicial de una palabra dictada); y que (tercera hipótesis) conocer el nombre de las letras hace que sea más fácil para los niños identificar la letra inicial que completa la escritura de una palabra dada.

En cuanto a estas hipótesis, encontramos que tener la información del nombre de la letra no implicaba poder usarla para completar o escribir palabras, y que incluso pudiera usar esa información en una tarea no implicaba poder usarla en otra; en todo caso, la utilidad de este conocimiento estuvo, como lo hemos ya dicho, condicionado a las conceptualizaciones de los niños sobre el sistema de escritura, y en menor medida con el contexto de motivación de la palabra a escribir o a completar. Así que los niños con escrituras S-A presentaron escrituras de la sílaba inicial convencionales $(\mathrm{CV})$ en contextos de contraste, y en mayor medida en las sílabas no motivadas.

Podemos concluir, entonces que si la coincidencia entre el contexto motivado y el nombre de la letra, por un lado, favorecía la escritura usando la consonante respectiva, por otro lado, perjudicaba la posibilidad del registro más avanzado conceptualmente (CV). Las respuestas de los niños con escrituras S-A apuntan a señalar que no fue el conocimiento del nombre de la letra y su coincidencia con el contexto silábico lo que hizo posible la escritura convencional, sino que el nivel conceptual condicionó el uso que estos niños hicieron de la información del nombre de la letra.

Nuestros datos sugieren que los niños con escrituras SCPS, las sílabas motivadas representaron una posibilidad real de elegir la consonante, mientras que las no motivadas les hacían elegir la vocal que componía la sílaba. Los datos también sugieren que conocer el nombre convencional una letra no fue el factor determinante para garantizar la pertinencia de la respuesta. Esto se debe a que, aunque observamos comportamientos de respuesta que variaban según la motivación fonológica, el denominador común fue el nivel de escritura, ya que, en su mayoría, los niños conocían el nombre de las letras.

\section{plupais}

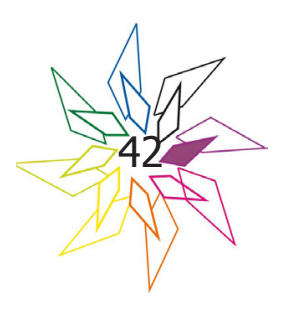


Finalmente, la última hipótesis de este trabajo fue que el nivel de escritura condiciona la utilidad que el conocimiento del nombre de la letra representa para el niño cuando escribe o completa palabras escritas. A lo largo de exposición de nuestro trabajo se hizo evidente la influencia de la reflexión que el niño tiene en la escritura sobre el uso de datos contextuales y lingüísticos en sus producciones escritas o para completar palabras.

En la última etapa del análisis de datos cuando realizamos la correlación entre identificar, escribir y completar palabras, observamos que la posibilidad de coordinar información y contextos y tener respuestas más consistentes también ocurría de acuerdo con el nivel de conceptualización.

Para terminar, quisiéramos apuntar que los hallazgos encontrados en este estudio nos permitieron confirmar que, antes de escribir convencionalmente, los niños tienen información sobre qué son las letras y cómo se llaman. Sin embargo, la utilidad de esta información parece diferir dependiendo de la tarea que se les pide que hagan (escribir o completar una palabra), los contextos silábicos a los que sirven y, sobre todo, la conceptualización que han construido sobre el sistema de escritura. En otras palabras, los niños de edades similares pueden presentar más o menos información sobre el sistema de escritura, pero la posibilidad de usar esta información está condicionada por la conceptualización que ya han construido sobre este sistema.

\section{REFERENCIAS}

ALVARADO, M. Conciencia fonológica y escritura de niños preescolares: la posibilidad de omitir el primer segmento (Tesis de Maestria). Universidad Autónoma de Querétaro, México. 1997.

CANO, S., VERNON, S. Lo que los niños saben de las letras antes de comenzar a escribir convencionalmente. (Tesis de Maestria). Universidad Autónoma de Querétaro, México. 2008.

CARDOSO-MARTINS, C., BATISTA, A. C. E. O conhecimento do nome das letras e o desenvolvimento da escrita: Evidência de crianças falantes do português. Psicologia: Reflexão \& Crítica, 18 (3), 330-336. 2005.

CARDOSO-MARTINS, C., RESENDE, S. M., RODRIGUES, L. A. Letter name knowledge and the ability to learn to read by processing letter-phoneme relations in words: Evidence from Brazilian Portuguese-speaking children. Reading and Writing, 15, 409-432. 2002.

EHRI, L. Sources of difficulty in learning to spell and read. In D. Routh (Org.), Advances in developmental and behavioral pediatrics (p. 121-195). Hillsdale: Erlbaum. 1986

\section{plurais}


FERNÁNDEZ, N. y ALVARADO, M. El efecto del rasgo de continuidad en la identificación de palabras escritas, Revista de Educación y Desarrollo, 32, pp, 29-39. 2015.

FERREIRO, E, TEBEROSKY, A. Los sistemas de escritura en el desarrollo del niño, México: Siglo XXI. 1979.

FERREIRO, E. Entre la sílaba oral y la palabra escrita. Infancia y Aprendizaje, 89, 25-37. 2000.

FERREIRO, E., GÓMEZ Palacios, M., \& Colaboradores. Análisis de las Perturbaciones en el Proceso de Aprendizaje de la Lecto-Escritura. In Fascículo 4: Las Relaciones entre el Texto como Totalidad- y sus Partes. México. 1982.

FERREIRO, E. Las unidades de lo oral y las unidades de lo escrito. Archivos de Ciencias de la Educación, 1 (1 cuarta época)(Universidad de La Plata, Argentina), 195-230. 2007.

MARQUES, L. F. Estrutura silábica do português do Brasil: uma análise tipológica. São Paulo, SP, Brasil: Universidade São Paulo. 2008.

POLLO, T., KESSLER, B., TREIMAN, R. Vowels, syllables, and letter names: differences between young children s spelling in English and Portuguese. Experimental Child Psychology, 92, 161-181. 2005.

POLLO, T., TREIMAN, R., KESSLER, B. Uma revisão crítica de três perspectivas sobre o desenvolvimento da escrita. Estudos de Psicologia (Campinas), 32(3), 449-459. 2015.

QUARESMA, P., PINHO, A. Análise de Frequências da Língua Portuguesa. In Livro de Actas da Conferência Ibero-Americana InterTIC. p. 267-272. Porto: Portugal, 2007.

READ, C., TREIMAN, R. Children's invented spelling: What we have learned in forty years. In R. C. Berwick (Org.), Rich languages from poor inputs (p. 197-211). New York: Oxford University Press. 2013

TREIMAN, R. Use of consonant letter names in beginning spelling. Developmental Psychology, 30 (4), 567-580. 1994.

TREIMAN, R. Why spelling? The benefits of incorporating spelling into beginning reading instruction. In L. C. Ehri (Org.), Word recognition in beginning (p. 289-313). Mahwah, N.J: Erlbaum. 1998.

TREIMAN, R., Bowman, M. Are young children logographic readers and spelleres? Scientific Studies of Reading, 12, 185-202. 2008.

\section{plurais}


TREIMAN, R., Rodríguez, K. Young children use letter names in learning to read words. Psychological Science, 10, 334-338. 1999.

TREIMAN, R., HULSLANDERB, J., OLSON, R., WILLCUTT, E., BYRNEC B., KESSLERA. B. The Unique Role of Early Spelling in the Prediction of Later Literacy Performance. Scientific Studies of Reading, 23, 437-444. 2019.

TREIMAN, R., POLLO, T., CARDOSO-MARTINS, C., KESSLER, B. Do young children spell words syllabically? Evidence from learners of Brazilian Portuguese. J Exp Child Psychol, 116 (4), 873-890. 2013.

TREIMAN, R., SOTAK, L., BOWMAN, M. The role of letter names and letter sounds in connecting print and speech. Memory \& Cognition, 29, 860-873. 2001.

VERNON, S. La relación entre la conciencia fonológica y los niveles de conceptualización de la escritura (Tesis de doutorado). DIE, CINVESTAV-IPN, México. 1997.

VERNON, S. La identificación de letras, la conciencia fonológica y el desarrollo de la escritura en niños preescolares. Apresentado em Memorias del 2o Simposio Internacional de "Lectura y Vida", Buenos Aires: IRA. 2001

VERNON, S. La relación entre la conciencia fonológica y los niveles de conceptualización de la escritura (Tesis de doctorado). DIE, CINVESTAV-IPN, México. 2005.

ZAMUDIO, C. Influencia de la escritura alfabética en la segmentación de sonidos vocálicos y consonánticos. Lectura y Vida, 29 (1), 10-21. 2008.

Recebido em: 07 de julho de 2020.

Inserido em: 15 de outubro de 2020.

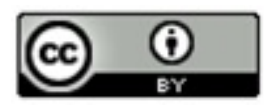

Esta obra está licenciada com uma Licença Creative Commons Atribuição 4.0 Internacional.

\section{plurais}

